\section{MODELO DE PROGRAMACIÓN LINEAL ENTERA MIXTA PARA EL PLANEAMIENTO DE LAS IMPORTACIONES EN RÉGIMEN ADUANERO DEFINITIVO}

Recepción: Noviembre de 2005 / Aceptación: Diciembre de 2005
(1) Christian Cornejo Sánchez

(2) Miguel Mejía Puente

\section{RESUMEN}

El presente trabajo trata sobre la aplicación de la Programación Lineal Entera Mixta (PLEM) como herramienta para la planificación de las importaciones, en el contexto de una empresa dedicada a la manufactura y venta de componentes de aceros. Se construye un modelo matemático que se adapte a las características de los procesos de importación de la empresa y cuyo objetivo será el de decidir cuál es el plan de importaciones con régimen aduanero definitivo que tiene costos totales mínimos. En esta primera parte, se formula el modelo matemático.

Palabras Clave: Programación lineal entera mixta, importación.

THE MIXED INTEGER LINEAR PROGRAMMING MODEL FOR THE IMPORT PLANNING IN DEFINITIVE CUSTOMS REGIME

\section{ABSTRACT}

The article deals in the application of the Mixed Integer Linear Programming (MILP) as a tool for the import planning in the context of a company dealing in the manufacturing and sale of steel components A mathematical model is built to be adapted for the characteristics of the company import processes and whose objective is to decide which import plan having definitive customs regimen has the minimum total costs. The mathematical pattern is formulated in this first part.

Key words: Mixed whole linear programming, import.

(1) Ingeniero Industrial. Profesor del Departamento de Ingeniería Industrial, PUCP E-mail: ccornejo@pucp.edu.pe

(2) Ingeniero Industrial. Profesor del Departamento de Ingeniería Industrial, PUCP. Doctorando en Ingeniería Industrial, UNMSM

E-mail: miguel.mejia@pucp.edu.pe

\section{INTRODUCCIÓN}

Este trabajo presenta una aplicación de la investigación de operaciones, utilizando la técnica de Programación Lineal Entera Mixta, con el objetivo de definir el planeamiento de las importaciones, en régimen aduanero definitivo, en una empresa que importa partes y piezas para producción y posterior comercialización.

\section{ALCANCES Y LIMITACIONES DEL MODELO MATEMÁtiCO}

El planeamiento de las importaciones consiste principalmente en la selección de los proveedores, la definición del tamaño del lote de la importación, la determinación del período entre importaciones, la selección de la modalidad de transporte y la reducción del costo de oportunidad que genera el dinero invertido en los tiempos prolongados, que caracterizan a los procesos y actividades de importación.

El régimen aduanero definitivo para importaciones es aquél que permite ingresar legalmente mercancías que provienen del exterior, para ser destinadas para el consumo en el mercado interno.

La importancia de este régimen no sólo está en su efecto recaudador, sino también, porque permite el ingreso de mercancías que la industria local puede transformar en productos, con un mayor valor agregado, para consumo nacional o posterior exportación.

La estructura general de costos para partes importadas, en este régimen, es el siguiente:

- Valor FOB + flete + seguro.

- Ad/Valorem, cuya base imponible, según el Acuerdo del Valor de la Organización Mundial de Comercio, es la suma del valor FOB, más el flete y el seguro.

- Gastos de desaduanaje.

- Comisión de la agencia de aduana.

\section{Asunciones}

Respecto a la operatividad de las importaciones, los siguientes supuestos definen el escenario en el cual se desarrolló el presente trabajo:

1. El horizonte de tiempo es anual y se asume 30 días para el despacho de mercancías, el cual corresponde al tiempo de producción promedio del proveedor en el extranjero.

2. El monto del seguro se calculará, para cualquier caso, con la tasa de seguro definida en el arancel del producto. 
3. Según lo anterior, el monto del seguro no se incluirá como un costo adicional en la función objetivo, sólo servirá para definir la base imponible del ad/valorem y demás impuestos.

4. Las modalidades de transporte internacional, usadas para este modelo son: aéreo y marítimo.

5. En este trabajo no se consideran las sobretasas arancelarias, cuyo tratamiento es similar al ad/ valórem, ni los derechos correctivos provisionales ad/valórem, por cuanto, los productos incluidos en el modelo no pertenecen a esas partidas arancelarias, ni los derechos específicos-sistema de franja de precios porque no son productos agropecuarios. Tampoco se trata de confecciones textiles, por lo tanto, no se aplican las salvaguardias de transición temporal.

6. Como el modelo es determinístico, no contempla el carácter aleatorio de la definición del color del canal de despacho aduanero (rojo, naranja, verde). El caso más costoso y con trámite más lento corresponde al canal rojo. Esta situación aleatoria se supera definiendo un monto promedio para los gastos de desaduanaje, incluido el monto del aforo físico; por tanto, asumimos un escenario pesimista para el modelo.

7. El escenario global del modelo es económico $u$ operativo, es decir, sólo se analizan los costos operativos vinculados a la importación. Por lo tanto, no consideraremos los gastos financieros por financiamiento.

8. El modelo se plantea para períodos entre importaciones, mensual, bimestral y trimestral. Sin embargo, es posible agregar otros períodos. La lógica del modelo es la misma.

9. Los resultados del modelo indicarán el período óptimo entre importaciones para cada proveedor, así como un tamaño uniforme para el lote de compra. Por ejemplo, si el período de importaciones para un origen k es trimestral, significa que cada tres meses se efectúa una importación desde ese origen $\mathrm{k}$, y que siempre el tamaño de lote de compra es el mismo.

10.El Artículo 17 de la Ley General de Aduanas, permite el pago de derechos arancelarios y demás tributos, según el estado físico de la mercancía (Valoración de mercancías dañadas). El estado físico de la mercadería que llega es una variable aleatoria. Como nuestro modelo es determinístico, se asumirá que la mercancía que la empresa importa siempre llega en buenas condiciones, y que el pago de derechos arancelarios y demás impuestos se realiza según este escenario.

11. El modelo está elaborado en el escenario que define la figura de "Contrato de Mandato" -Artículo 99 de la Ley General de Aduanas- en la cual la empresa importadora, faculta al agente de aduana a efectuar, en su representación, los trámites de desaduanaje. La agencia de aduana cobra una comisión y los gastos operativos por el servicio, lo que se incluirá en el modelo.

Teoría general de la programación lineal entera

Un modelo de programación lineal entera es aquel en el cual algunas de las variables o todas, son números enteros no negativos.

En las situaciones reales, con frecuencia, el analista se enfrenta a "decisiones sí o no", las que pueden representarse con variable denominadas binarias, por ejemplo 0 y 1 . Así la k-ésima decisión sí o no puede representarse por $X_{k}$, tal que:

$$
X_{k}=\left\{\begin{array}{l}
1 ; \text { si la decisión } k \text { es sí. } \\
0 ; \text { si la decisión } k \text { es no. }
\end{array}\right.
$$

Cuando sólo es necesario que algunas de las variables sean enteras y el resto continuas, el modelo recibe el nombre de problema de Programación Lineal Entera Mixta. Dentro de esta clasificación, se incluyen aquellos modelos que, además de tener variables enteras no negativas y variables continuas, tienen también variables binarias.

\section{MODELACIÓN}

\section{Variables de decisión}

Uptijk: Unidades mensuales de la pieza i, del producto j, origen k. Recibe los valores óptimos de las unidades de cada pieza ijk, con período entre importaciones mensual, bimestral o trimestral, en las dos modalidades de transporte elegidas: aérea y marítima.

Fptk: Variable para el costo del flete de las importaciones desde el origen $\mathrm{k}$. Recibe el valor del flete aéreo o marítimo, para las importaciones efectuadas desde el origen $\mathrm{k}$.

Blptk: Variable para el valor de aduanas de las importaciones desde el origen k. Recibe el valor de aduanas (CIF) de la importación efectuada desde el origen $\mathrm{k}$, en cualquiera de las modalidades de transporte y con un período de importaciones determinado.

AVIptk: Variable para el ad/valórem, IGV e IPM de las importaciones desde el origen $\mathrm{k}$. Recibe el ad/valorem, IGV e IPM de las importaciones desde el origen $k$, en cualquiera de las modalidades de transporte y con un período de importaciones determinado.

COptk:Variable para el costo de oportunidad de las importaciones desde el origen k. Recibe el costo de oportunidad de las importaciones desde el origen $\mathrm{k}$, en cualquiera de las moda- 
lidades de transporte y con un período de importaciones determinado.

Cptk: Costo FCA o FOB + seguro + flete aéreo o marítimo de todas las piezas importadas $\mathrm{i}$, del producto j, origen k. Recibe el valor de aduanas de las variables BIPtk, respectivamente.

Para todas las variables anteriores, se tiene que: $t=1$, importaciones por vía aérea; $t=2$, importaciones por vía marítima; y $p=1, p=2, p=3$ indican período entre importaciones: mensual, bimestral y trimestral, respectivamente.

\section{Parámetros}

$\tau \mathrm{rd} / \mathrm{vi}$ : Tasa aduanera, aplicada a la base imponible $\mathrm{del}$ ad/valórem, según la partida arancelaria de la pieza importada i.

IGV: Valor de la tasa del IGV.

IPM: Valor de la tasa del IPM.

$\mu$ : $\quad$ Monto fijo que cobra la comisión de la agencia de aduana, cuando el monto CIF es menor a $\alpha$

$\rho: \quad$ Tasa que cobra la agencia de aduana por su servicio, aplicable al valor de aduana de la mercancía.

$\alpha$ : $\quad$ Monto CIF, a partir del cual la comisión de agencia de aduana es variable e igual a $\rho^{*}$ (monto CIF).

$\gamma$ : $\quad$ Promedio de los gastos de desaduanaje. No incluye la comisión de la agencia de aduana.

$1 \%$ : Costo de oportunidad del capital.

TP: Días de producción del proveedor extranjero, previos a la fecha de despacho. Se definió igual a 30 días para todos los proveedores del extranjero.

TTk: Días de transporte internacional para la mercadería importada desde el origen $\mathrm{k}$.

TDk: Días que demora el desaduanaje de la mercadería, cuyo origen es $\mathrm{k}$.

\section{Función objetivo}

Minimizar los costos de la importación.

$\sum_{\mathrm{k}=1}^{\mathrm{s}} \sum_{\mathrm{j}=1}^{\mathrm{m}} \sum_{\mathrm{i}=1}^{\mathrm{n}} \mathrm{cexw} i j k^{*} \mathrm{Uptjk}+\sum_{\mathrm{k}=1}^{\mathrm{s}} \sum_{\mathrm{j}=1}^{\mathrm{m}} \sum_{\mathrm{i}=1}^{\mathrm{n}} \mathrm{cfijk}^{*} \mathrm{Uptjk}+$

$12 * \sum_{\mathrm{k}=1}^{\mathrm{s}} \mathrm{F} 1 t k+6 * \sum_{\mathrm{k}=1}^{\mathrm{s}} \mathrm{F} 2 t k+4 * \sum_{\mathrm{k}=1}^{\mathrm{s}} \mathrm{F} 3 t k+$

$\sum_{\mathrm{k}=1}^{\mathrm{s}} \sum_{\mathrm{j}=1}^{\mathrm{m}} \sum_{\mathrm{i}=1}^{\mathrm{n}} \theta i * \operatorname{cexwijk} * \mathrm{U} p t j k+\sum_{\mathrm{k}=1}^{\mathrm{s}} \theta i * \eta t k * \mathrm{Fptk}+$

$\sum_{\mathrm{k}=1}^{\mathrm{s}} \sum_{\mathrm{j}=1}^{\mathrm{m}} \sum_{\mathrm{i}=1}^{\mathrm{n}} \theta i * \mathrm{cft} t i j k * \mathrm{U} t j k+12 * \sum_{\mathrm{k}=1}^{\mathrm{s}} \mathrm{AVI} l t k+\mathrm{t}$

$$
-6 * \sum_{\mathrm{k}=1}^{\mathrm{s}} \operatorname{AVI} 2 t k+4 * \sum_{\mathrm{k}=1}^{\mathrm{s}} \mathrm{AVI} 3 t k+\sum_{\mathrm{p}=1}^{3} \gamma^{*}(1-\mathrm{zptk})+
$$

$\sum_{\mathrm{p}=1}^{3}\left(\mathrm{w} p t 1 k * \mu+{ }_{w p t 2 k} * \mu+w p t 3 k * \rho * N-\mu *_{z p t k}\right)+$

$12^{*} \sum_{\mathrm{k}=1}^{\mathrm{s}} \mathrm{CO} 1 \mathrm{tk}+6^{*} \sum_{\mathrm{k}=1}^{\mathrm{s}} \mathrm{CO} 2 t \mathrm{k}+4^{*} \sum_{\mathrm{k}=1}^{\mathrm{s}} \mathrm{CO} 3 t \mathrm{k}$

Donde:

cexwijk:Costo unitario EXW de la pieza i, del producto j, origen $\mathrm{k}$.

cftijk: Si $t=1$, entonces el costo unitario es FCA; si $t=2$, entonces el costo unitario es FOB; de la pieza i, del producto j, origen $\mathrm{k}$.

$\eta$ tk: $\quad$ Porcentaje que representa, del flete internacional, los gastos al FCA o FOB, cuando la importación es desde el origen $\mathrm{k} ; \mathrm{k}=1,2,3 \ldots \mathrm{p}$

$\sum_{\mathrm{k}=1}^{\mathrm{s}} \eta t k^{*} \mathrm{~F} p t k$ son los gastos al FCA o FOB

Los dígitos que acompañan a cada variable en la expresión mostrada, indican la cantidad de períodos en un año.

\section{Restricciones}

Flete aéreo y flete marítimo:

Fptk $\geq \phi \mathrm{a}_{k} * \sum_{\mathrm{j}=1}^{\mathrm{m}} \sum_{\mathrm{i}=1}^{\mathrm{n}} \boldsymbol{\omega}_{i j k} * \mathrm{U} p t i j k \quad \mathrm{k}=0,1,2, \ldots \mathrm{s} ; \mathrm{t}=1$

$\mathrm{F} p t k \geq \phi \mathrm{a} k^{*} \sum_{\mathrm{j}=1}^{\mathrm{m}} \sum_{\mathrm{i}=1}^{\mathrm{n}} \beta^{*}\left(\mathcal{U}_{i j k} / 100^{\wedge} 3\right)^{*} \mathrm{U} p t i j k \quad \mathrm{k}=0,1,2, \ldots \mathrm{s} ; \mathrm{t}=1$

Fptk $\geq \phi_{\mathrm{m}_{k}} \sum_{\mathrm{j}=1}^{\mathrm{m}} \sum_{\mathrm{i}=1}^{\mathrm{n}} \boldsymbol{V}_{i j k}{ }^{*} \mathrm{U} p \mathrm{tijk} \quad \mathrm{k}=0,1,2, \ldots \mathrm{s} ; \mathrm{t}=2$

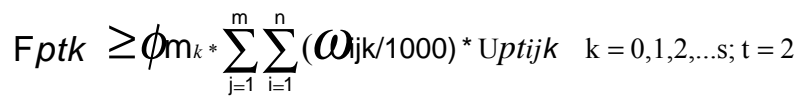

Donde:

vijk: Volumen unitario en $\mathrm{cm}^{3}$ de la pieza i, del producto $\mathrm{j}$, importada del origen $\mathrm{k}$.

$\omega i j k$ : Peso unitario en kilogramos de la pieza i, del producto j, importada del origen $\mathrm{k}$.

$\beta$ : $\quad$ Factor peso-volumen igual a 1000/6

$\phi a k$ : Costo unitario del flete internacional, en unidades monetarias por cada kilogramo, para mercancía importada por vía aérea, desde el origen $\mathrm{k}$.

$\phi \mathrm{m} k$ : Costo unitario del flete internacional, en unidades monetarias, por cada tonelada o metro cúbico, para mercancía importada por vía marítima, desde el origen $\mathrm{k}$. 
Ad/valórem:

AVIptk $\geq$ Expresión ad/valorem más IGV e IPM, según el criterio del peso total, ya sea en el caso aéreo o en el caso marítimo.

AVIptk $\geq$ Expresión ad/valorem más IGV e IPM, según el criterio del peso equivalente total, en el caso aéreo o según el criterio de volumen total en el caso marítimo.

Gastos de desaduanaje:

zptk $\leq \mathrm{M}^{*} \mathrm{vptk}$

$\mathrm{CM} t \mathrm{tk} \leq \mathrm{M}^{*}(1-\mathrm{vptk})$

1 - zptk $\leq M^{*}$ Cptk

Comisión de las agencias de aduana:

Blptk $\geq$ Expresión de la base imponible o valor de aduanas, según el criterio del peso total, ya sea en el caso aéreo o en el caso marítimo.

Blptk $\geq$ Expresión de la base imponible o valor de aduanas, según el criterio del peso equivalente total, en el caso aéreo o según el criterio del volumen total en el caso marítimo.

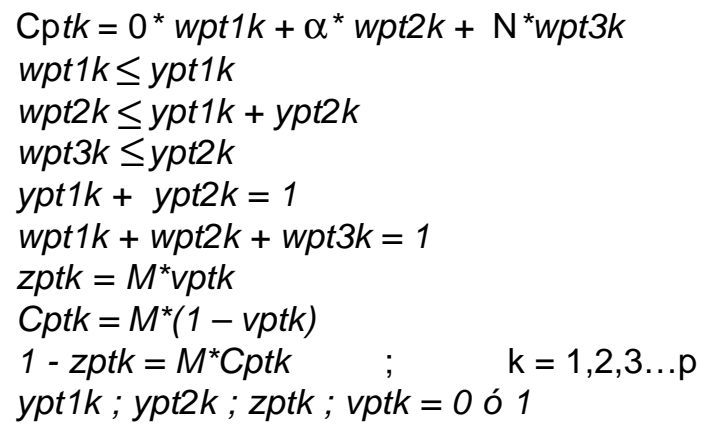

Donde:

$\mathrm{M}$ y $\mathrm{N}$ son números muy grandes.

wpt $1 k$; wpt2k; wpt3k $\geq 0$

vptk ; ypt1k ; ypt2k ; zptk = 0 ó $1 ; \mathrm{t}=1$ aéreo; $\mathrm{t}=2$ marítimo.

$p=1, p=2, p=3$ indican período entre importaciones: mensual, bimestral y trimestral, respectivamente.

El costo de oportunidad:

Sea A: Costo de adquisición de la mercadería importada Sea B: Costo del transporte internacional

Sea C: Costo derechos arancelarios

El costo de oportunidad de producción es:

$\mathrm{COP}=\left((1+l \%)^{\wedge(\mathrm{TP} / 360)}-1\right)^{\star}(\mathrm{A})$

El costo de oportunidad del transporte es:

$\mathrm{COT}=\left((1+1 \%)^{\wedge(T T K / 360)}-1\right)^{*}(\mathrm{~A}+\mathrm{B})$

El costo de oportunidad del desaduanaje es:

$\mathrm{COD}=\left((1+1 \%)^{\wedge(\mathrm{TDK} / 360)}-1\right)^{*}(\mathrm{~A}+\mathrm{B}+\mathrm{C})$
Para el costo de oportunidad, hasta que se agota el stock importado, utilizamos el siguiente modelo, definiendo a $C$ como: $D=A+B+C$.

Si el período entre importaciones es mensual tenemos: $\left((1+1 \%)^{\wedge(30 / 360)}-1\right)^{\star} \mathrm{D}$.

Si el período entre importaciones es bimestral tenemos: $\left((1+1 \%)^{\wedge(60 / 360)-1}\right)^{\star}\left((1 / 2)^{*} \mathrm{C}\right)+\left((1+1 \%)^{\wedge(30 / 360)}-1\right)^{*}\left((1 / 2)^{*} \mathrm{C}\right)$

COptk $\geq$ Expresión del costo de oportunidad, según el criterio del peso total, ya sea en el caso aéreo o en el caso marítimo.

COptk $\geq$ Expresión del costo de oportunidad, según el criterio del peso equivalente total, en el caso aéreo o según el criterio del volumen total en el caso marítimo.

Restricciones para los períodos excluyentes entre importaciones.

Para las importaciones con período mensual:

$\mathrm{U} 2$ tijk + U 3 tijk $\leq \mathrm{M}^{*}(\mathrm{x} 1$ tijk $)$

$\mathrm{U} 1$ tijk $^{\prime} \leq \mathrm{M}^{*}(1-\mathrm{x} 1 \mathrm{tijk})$

$\mathrm{x} 1$ tijk $=0$ ó 1

Para las importaciones con período bimestral:

$\mathrm{U} 1$ tijk + U3tijk $\leq \mathrm{M}^{*}(\mathrm{x} 2 t i j k)$

$\mathrm{U} 2$ tijk $\leq \mathrm{M}^{*}(1-\mathrm{x} 2$ tijk $)$

$\mathrm{x} 2$ tijk $=0$ ó 1

Para las importaciones con período trimestral:

$\mathrm{U} 1$ tijk + $\mathrm{U} 2 t i j k \leq \mathrm{M}^{*}(\mathrm{x} 3 \mathrm{tijk})$

U3tijk $\leq \mathrm{M}^{*}(1-\mathrm{x} 3$ tijk $)$

$\mathrm{x} 3$ tijk = 0 ó 1

Restricciones de demanda.

$$
\begin{aligned}
& 12 * \sum_{\mathrm{k}=1}^{\mathrm{s}} \mathrm{U} 1 t 1 k+6 * \sum_{\mathrm{k}=1}^{\mathrm{s}} \mathrm{U} 2 t 1 k+4 * \sum_{\mathrm{k}=1}^{\mathrm{s}} \mathrm{U} 3 t 1 k \geq \mathrm{a} i j * \mathrm{D} i j \\
& \mathrm{i}=1,2, \ldots \mathrm{n} ; \mathrm{j}=1,2, \ldots \mathrm{m}
\end{aligned}
$$

Donde:

aij es el parámetro cantidad de piezas ique se necesitan en el producto $j$.

Djes el parámetro demanda del producto $j$.

Rango de resistencia

Uptijk $\geq 0$ y enteras

Fptk $\geq 0$

Blptk $\geq 0$

AVIptk $\geq 0$

COptk $\geq 0$

Cptk $\geq 0$

$p=1,2,3$ 


\section{CONCLUSIONES}

Se ha elaborado el modelo según los supuestos definidos, los cuales surgen de la operatividad real de una importación, así como las restricciones que impone la Ley General de Aduanas.

El modelo propuesto muestra la posibilidad de expresar la complejidad del comportamiento de los costos en importaciones con régimen aduanero definitivo. Así, costos fijos, variables y de comportamiento mixto se expresaron en un modelo lineal, por medio de las técnicas del problema de costo fijo de programación entera, minimización del máximo progreso y la programación lineal entera por partes.

Superado el problema de expresar los costos de importación en un formato lineal, el modelo evaluará y determinará cuál es la solución que minimiza los costos de un plan de importación. De esta manera, sus resultados permitirán realizar una planificación de las importaciones que indiquen modalidad de transporte, tiempo óptimo entre importaciones, tamaño de lote y selección de proveedores. En un próximo trabajo, mostraremos los resultados para una serie de datos.

\section{BIBLIOGRAFÍA}

1. Cosío Jara, Fernando. (1997). Comentarios a la Ley General de Aduanas. Primera Edición, Librerías y Ediciones Jurídicas, Lima, Perú.

2. Domínguez Machuca, José; García, Santiago; Domínguez Machuca, Miguel; Ruiz, Antonio; Álvarez, María. (1995). Dirección de Operaciones. Aspectos Tácticos y Operativos en la Producción y los Servicios. Primera Edición. McGraw-Hill, España.

3. Hillier, Frederick S.; Lieberman, Gerald J. (1994). Introducción a la Investigación de Operaciones. Cuarta edición, McGraw-Hill. México.

4. Winston, Wayne L. (1994). Investigación de Operaciones. Aplicaciones y Algoritmos. Primera edición, Grupo Editorial Iberoamérica. México. 